\title{
Haptic-Multimodal Flight Control System Update
}

\author{
Kenneth H. Goodrich ${ }^{1}$ and Paul C. Schutte ${ }^{2}$ \\ NASA Langley Research Center, Hampton, VA, 23681 \\ Ralph A. Williams ${ }^{3}$ \\ Analytical Mechanics Associates, Hampton, VA, 23666
}

\begin{abstract}
The rapidly advancing capabilities of autonomous aircraft suggest a future where many of the responsibilities of today's pilot transition to the vehicle, transforming the pilot's job into something akin to driving a car or simply being a passenger. Notionally, this transition will reduce the specialized skills, training, and attention required of the human user while improving safety and performance. However, our experience with highly automated aircraft highlights many challenges to this transition including: lack of automation resilience; adverse human-automation interaction under stress; and the difficulty of developing certification standards and methods of compliance for complex systems performing critical functions traditionally performed by the pilot (e.g., sense and avoid vs. see and avoid). Recognizing these opportunities and realities, researchers at NASA Langley are developing a haptic-multimodal flight control (HFC) system concept that can serve as a bridge between today's state of the art aircraft that are highly automated but have little autonomy and can only be operated safely by highly trained experts (i.e., pilots) to a future in which nonexperts (e.g., drivers) can safely and reliably use autonomous aircraft to perform a variety of missions. This paper reviews the motivation and theoretical basis of the HFC system, describes its current state of development, and presents results from two pilot-in-the-loop simulation studies. These preliminary studies suggest the HFC reshapes human-automation interaction in a way well-suited to revolutionary ease-of-use.
\end{abstract}

$\begin{array}{ll} & \\ \text { cHAA } & =\text { contemporary highly automated aircraft } \\ \text { CHR } & =\text { Cooper-Harper Rating } \\ \text { CTTM } & =\text { control and tactical trajectory management } \\ \text { FBW } & =\text { fly-by-wire } \\ \text { HAI } & =\text { human-automation interaction } \\ \text { HFC } & =\text { haptic-multimodal flight control } \\ \text { NAS } & =\text { National Airspace System } \\ \text { NFD } & =\text { Naturalistic Flight Deck } \\ \text { P2P } & =\text { Point to Program } \\ \text { PAV } & =\text { Personal Air Vehicle } \\ \text { PFD } & =\text { primary flight display } \\ \text { UAS } & =\text { unmanned aircraft system } \\ \text { SAGAT } & =\text { Situation Awareness Global Assessment Technique } \\ \text { TND } & =\text { tactical navigation display }\end{array}$

\section{Introduction - A Case for Incremental Revolution}

common belief is that easy-to-use Personal Air Vehicles (PAVs) can only be achieved through full autonomy. Often, the underlying reasoning is that the human simply cannot be trusted to do the right thing. Just looking at drivers on the road is enough to confirm this belief. Or is it? While we've all seen drivers do crazy things and probably made a few blunders ourselves, the bottom line is that we find cars, operated by the public, safe enough

\footnotetext{
${ }_{1}^{1}$ Aerospace Technologist, Dynamic Systems and Guidance Branch, Mail Stop 308, AIAA Senior Member.

${ }^{2}$ Aerospace Technologist, Crew Systems and Aviation Operations Branch, Mail Stop 152.

${ }^{3}$ Senior Analyst, Dynamic Systems and Guidance Branch, Mail Stop 308.
} 
that we have made them an integral part of our daily lives. We rarely give safety a second thought when considering a car trip. We would all like safer cars and are willing to spend a little more to improve safety, but if people were told that the roads were unsafe and that they could not use automobiles anymore until we perfected the autonomous vehicle, people would say "Forget that!" and get in their cars anyway. If PAVs could achieve similar ease-of-use, utility, economy, and safety as cars, are we not likely to similarly integrate them into our lifestyles, regardless of the level of autonomy?

From practical experience, we are much more comfortable with risk when there is a human in control who can respond to novel situations. In all likelihood, a fully-automated PAV would be subject to a much higher standard of safety than a piloted one. This added scrutiny would require additional time and money to satisfy. But how much time and money will be required, and will the benefit be worth the expense? Assessing the time and costs to bridge the technology gap between the current state of the art and full-autonomy within even a rough order-of-magnitude is problematic given the required technology breakthroughs. The most fundamental breakthroughs are of course, technological substitutes for the perception, world knowledge, general intelligence, and creative thinking that today's pilot brings to the cockpit. As discussed later in the paper, we believe that current, highly automated aircraft (manned or unmanned) and experimental, fully-autonomous cars and aircraft give the appearance of being much closer to having the necessary, human-like capabilities than they actually are. While current and emerging automation often exceeds human performance in terms of isolated engineering criteria, it tends to be inherently brittle to even small changes in the task or environment ${ }^{1}$. Despite amazing progress in the development of automated vehicles, we still have a long way to go.

A potentially more challenging problem for aviation applications involving inherent risk to humans (e.g., involving human cargo) is certification. The current regulatory framework is predicated on the presence of a human in the system, and changing this situation will be a long and contentious process. Current activities to substitute "sense and avoid" for "see and avoid" to enable co-mingled manned and unmanned aircraft system (UAS) operations illustrate the difficulties of this task and paint a daunting picture for any business expecting a return on investment in the near term. On top of these technical and regulatory challenges are societal challenges such as public trust and liability. 2010's rumors about electronics or software causing uncommanded automobile accelerations where the driver could not intervene foreshadow possible public concerns.

Because of these factors, we believe the most promising path, indeed perhaps the only practical path, to PAVs retains the human in the pilot's seat. This approach relies primarily on a restructuring of the Human/Automation Interaction (HAI) experience rather than a technological breakthrough. We believe that this can be achieved with a few simple technological advances, using existing technology. This new structure, rather than replacing the human, more fully supports them, enhancing their strengths and compensating for their weaknesses. At the same time the restructuring will create a framework in which emerging and future technologies can be introduced into the flight deck in a managed, incremental process. Initially, new technologies could be used in non-critical roles and later, once operational experience and certification criteria are in place, elevated to more critical uses. Through this process, we may one day find ourselves with intelligent PAVs that do not require our interaction, and we will have enjoyed the benefits of personal air travel along the way.

But how should the HAI and supporting interface be re-engineered to support these goals? Additionally, will pilots perceive enough benefit in terms of safety and ease-of-use, during flight and training, from early implementations (e.g., retrofit) to begin and sustain a process of evolution that results in a true revolution? Presently, we have only partial answers to these questions. This paper provides a review and update on one of our concepts: a variable autonomy, haptic-multimodal flight control (HFC) system. The term "haptic" is used here to refer to the use of touch, force, and motion to underlie the HAI. The HFC is intended to be a component of an overall flight $\operatorname{deck}^{2}$ and provides integrated support for control and tactical trajectory management (CTTM) tasks. These tasks include, guidance, navigation, and control; formulation of trajectories and maneuvers satisfying nearterm objectives and constraints; and tactical (i.e., time-constrained) decision making in potentially dynamic situations. Examples of tactical decision making include deciding whether to continue or abort an approach; whether to accept a proposed clearance; how to reroute around traffic or local weather; and how to get on the ground safely in the event of a fire. In brief, CTTM can be thought of as the "aviate task" and time-pressured portions of the "navigate task" of the familiar "aviate, navigate, and communicate" prioritization scheme ${ }^{3}$. CTTM is central to safe and effective flight and breakdowns within CTTM, typically loss-of-control or/and poor tactical decision making, are causal or contributing factors in a large majority of aviation accidents ${ }^{4,5}$.

In the remainder of the paper we describe the expected requirements for PAV ease-of-use, the current state of the art in aviation and experimental autonomous vehicles. Next we describe the design concept and functionality of the HFC. We then summarize the results from two pilot in the loop simulation studies supporting the expected 
human-automation interaction benefits. We conclude with a summary and brief discussion of future efforts needed to enable PAVs.

We use the term "pilot" throughout the paper to broadly refer to the human presence in the human-aircraft system and it may, particularly when referring to present day operations, reflect multiple human actors (e.g., a twopilot crew and ground-based dispatcher). Similarly, when the term "pilot" is used to refer to an expert with a high level of preparation and training, this usage will be specifically noted. Also, while concepts of the HFC can be applied to unmanned aircraft, development to date has focused on on-board pilots and this focus is reflected in the current paper.

\section{Background}

\section{A. Personal Air Vehicle Requirements}

The expected operational characteristics and piloting requirements for PAVs are summarized in this section. The reader should recognize that given the notional nature of PAVs at this time, these characteristics are more illustrative than prescriptive. PAVs are expected to be light-aircraft carrying 1-10 people, including a pilot. Configurations may range from simple, single-engine piston aircraft up to very light jets. In the future, operations from small urban and suburban airfields may require precise, short or vertical takeoff and landing operations ${ }^{6}$. Flight in (near) all weather conditions is required to support reliable transportation. Early PAVs will have to operate in the National Airspace System (NAS) according to current and emerging rules such as being developed for the "NextGen" NAS ${ }^{7}$. Longer term, widespread adoption of PAVs would result in air traffic densities much greater than envisioned for NextGen and separation intervals in low-visibility on the order of 30 seconds $(\sim 0.5 \mathrm{~nm})$ may be needed in the vicinity of airports for adequate runway throughput. Propulsion systems are assumed to be controllable by something like a single-lever power controller with a full-authority, inner-loop engine controller. Loss of power is expected to be improbable, but something that needs to be considered within the context of CTTM. In-flight recovery systems such as a whole-aircraft parachute may be present, but they should be given no credit as a means of reducing the certitude of other systems.

Single-pilot operations are the norm for PAVs with ratings for non-commercial (i.e., private pilots) and commercial (e.g., air-taxi pilots) operations. Initially, these pilot ratings may fall under current pilot licensing requirements, albeit with equipage restrictions similar to a centerline-thrust restricted, multi-engine rating. With improved technology and operational experience, training required for near-all weather operation might be reduced to a level comparable to that of driver education (e.g., 24 hours of classroom (or on-line) instruction and 40 hours of supervised operation). This total is similar to the minimum hours to earn a visual flight rules, private-pilot license today may raise concern given the low completion rate of current student-pilots, which is around $20 \%{ }^{8}$. We believe the envisioned PAV rating would result in a much higher degree of transportation utility and result in completion rates similar to driver education. In addition to simplified initial training, another goal is to make piloting easy enough that flying solely to maintain proficiency is unnecessary for pilots flying once a month for transportation purposes. Finally, the risk of a serious accident should be no greater than travel by car. While it can be difficult to directly compare automotive and aviation safety statistics given the concentration of risk around takeoff and landing, we believe this goal will be achieved if the PAV fatal accident rate is an order-of-magnitude less than today's small aircraft. The current small aircraft fatal accident rate is 1.3 per 100,000 flight hours ${ }^{9}$ so a target rate of 1 per $1,000,000$ flight hours would be appropriate.

In the next section, we review current highly automated aircraft such as modern jets and many new small aircraft as well as experimental "driver-less" vehicles.

\section{B. Assessment of the State-of-the-Art}

How close is the current and emerging state of the art to enabling ease-of-use? In the next few paragraphs, we briefly review the capabilities of contemporary, highly-automated aircraft (cHAA) and experimental, autonomous vehicles with an eye toward the technological foundation they provide and insights they offer into HAI.

The terminology surrounding automated and autonomous vehicles can be confusing because terms like fullyautomated and autonomous are often used interchangeably but do not necessarily mean the same thing. For example the Global Hawk unmanned aircraft is often referred to as "autonomous" because it can conduct entire flights without interacting with a ground operator. However, a more appropriate description might be "fully-automated" rather than autonomous given that it follows pre-scripted mission plans, which can take up to four weeks to program and validate ${ }^{10}$, and is vulnerable to unforeseen but not improbable hazards like a truck parked on the runway. For practical purposes what is usually meant by an autonomous vehicle is a vehicle having a level of intelligence, i.e., the ability to make appropriate choices or decisions in an uncertain environment that is sufficient to perform 
complex missions without a human operator or mission-specific low-level programming defining all actions. This is the general meaning we associate with autonomy in the following discussion. We use fully-autonomous to indicate a vehicle needing no human interaction. The reader will also notice that the following discussion is largely silent on fully-automated aircraft like the Global Hawk. In general, these systems suffer from similar HAI challenges as piloted cHAA, often with more serious consequences given the great reliance on automation and separation between the operator and the vehicle ${ }^{11}$.

Contemporary highly-automated aircraft including modern transports, business jets, and many small aircraft now have sophisticated automation and can perform routine flights with little pilot interaction. But, it is important to recognize that this capability is far short of what is required for fully-automated, passenger carrying PAVs and is very different from the pilot playing a minor, easily eliminated role or requiring significantly less training, practice, or vigilance, than pilots of less automated aircraft. As Billings states,

The newest long-range airplanes can operate almost unassisted from shortly after takeoff in New York until coming to rest after a landing in Tokyo. The considerable psychomotor and cognitive skills of their human operators are hardly called upon unless some element of the automation fails or unanticipated environmental circumstances arise. But when the environment does not behave as expected, or when the very reliable machinery does not function correctly, we expect these human operators to do whatever is required to complete the mission safely. ${ }^{1}$

In effect, today's aircraft can perform the deterministic, routine and repetitive tasks that make up the most recognizable aspects of aircraft operation, but the most challenging tasks, including detecting and recovering from automation failures, are left up to the pilot. From a training and safety perspective, any task that a pilot may be called on to perform, even if only rarely, is still a task that they must be trained and prepared to perform. This required training does little to improve ease-of-use and introduces safety concerns due to potential lack of proficiency. The practice of automating routine tasks and leaving the more difficult leftovers to the pilot leaves a large gap to be crossed before fully autonomous PAVs can be realized. For piloted PAVs, operational experiences with cHAA provide insights into HAI constraints that must be addressed in the PAV's pilot interface; we discuss these issues in a latter section.

If current, conventional automation is a long way from enabling autonomous PAVs, a good question is: how quickly the technology from experimental, autonomous vehicles might close this gap? Research to expand the capabilities of fully-autonomous vehicles is receiving an enormous amount of attention and investment. The plummeting cost and increasing performance of sensors and computing hardware make this a fertile area for research. The most active area of research has probably been autonomous cars given the large number of applications, potential safety benefits, and visibility gained from a series of "Grand Challenges" sponsored by the Defense Advanced Research Project Agency (DARPA) between 2004 and 2007 ${ }^{12}$. In the most recent event, the Urban Challenge held in 2007, autonomous cars had to navigate through an urban environment including the ability to perceive and interact with other traffic. The winning entrant completed a 60 mile course with an average speed of $14 \mathrm{mph}$. Based in part on this work, Google recently launched a research program to develop autonomous cars ${ }^{13}$. Google's motivations include improved safety (targeting a 50\% reduction in fatalities), and the transformative impact self-driving cars would have on car-sharing. So far, Google's cars have logged over 140,000 miles without an accident, although always with a trained safety driver and led by a conventionally-driven car to ensure the route map is accurate and road conditions satisfactory. Comments from knowledgeable demonstration passengers, such as the adminstrator of the National Highway Traffic Safety and chairwoman of National Transportation Safety Board Chairwoman, find the technology impressive albeit with a long way to go before widespread, public use ${ }^{14}$.

The developments taking place on these vehicles and similar experimental flight vehicles ${ }^{15}$ are impressive and important advances that will improve the safety of ground and flight vehicles, both manned and unmanned. But, from the perspective of PAVs, what should be their role in the near future? What these activities most clearly demonstrate is that current technology is readily available to perform many of the low level tasks in the vehicle (e.g., staying in a lane, not following too closely, maintaining speed, staying on course) and can do so far better than a human operator. The performance areas that technology is reaching for and most likely to fail at (and indeed is most impressive when it comes close to performing) are those having to do with higher level, common sense, adaptive, and creative troubleshooting performance. Here, even the best technology is highly vulnerable. But we have those skills in abundance in the form of the human. Why do we feel that we must ignore those skills in the name of easeof-use and reliability? Yes, humans can and will make mistakes, but can we provide countermeasures for their errorproneness while utilizing their skills and still make flying easy and enjoyable to fly? We strongly believe that the answer is 'yes.' And no Grand Challenge is needed to prove it.

If current automation and emerging autonomous vehicle technologies are not close to bridging the gap to fullyautonomous PAVs, we are compelled to develop a more effective human-machine partnership. To do this, we take advantage of the insights provided by cHAA.

4

American Institute of Aeronautics and Astronautics 


\section{Where Humans Fail: Insights from Contemporary Highly Automated Aircraft}

While advanced avionics (e.g., flight management systems, auto-flight systems, electronically controlled propulsion, and advanced subsystems management) have greatly improved aviation safety, there are two important insights to be gleaned from their introduction and use. The first insight is the fallacy of reduced workload. As early as the 1990s, research showed that highly automated flight decks did not necessarily reduce crew workload. In fact, workload was reduced in already low periods but could be dramatically increased in high-workload periods ${ }^{1}$. This situation was due to automation that handles routine situations but generally gives up in uncertain, novel, or complicated situations. As far as workload is concerned, automation does not appear to be helping when it is needed most. Additionally, the presence of automation in the form of the flight management computer adds additional tasks (higher workload) just to program and remember how to operate the automation.

The second insight comes from the types of errors that humans make that currently lead to accidents. Figure 1 from Wiegmann and Rantanen ${ }^{16}$ shows the relative frequency of different classes of human error in commercial and general aviation respectively, giving insight to areas of human weaknesses. Wiegmann and Rantanen describe the error categories as follows:

Skill-based errors: Highly practiced behavior that occurs with little or no conscious thought. These "doing" errors frequently appear as breakdown in visual scan patterns, inadvertent activation/deactivation of switches, forgotten intentions, and omitted items in checklists.

Decision errors: These "thinking" errors represent conscious, goal-intended behavior that proceeds as designed, yet the plan proves inadequate or inappropriate for the situation. These errors typically manifest as poorly executed procedures, improper choices, or simply the misinterpretation and/or misuse of relevant information.

Perceptual errors: These errors arise when sensory input is degraded, as is often the case when flying at night, in poor weather, or in otherwise visually impoverished environments. Faced with acting on imperfect or incomplete information, aircrews run the risk of misjudging distances, altitude, and descent rates, as well as of responding incorrectly to a variety of visual/vestibular illusions.

Violations: Violations are divided into two sub-categories, routine and exceptional. Routine violations, often referred to as "bending the rules," tend to be habitual by nature and are often enabled by a system of supervision and management that tolerates such departures from the rules. Exceptional violations are isolated departures from authority, neither typical of the individual nor condoned by management.

What is important to note is that the errors are not being made in the areas emphasized in research into fully autonomous vehicles. They are being made in areas that machines are traditionally very proficient, i.e., precise, skill-based operations. Automation can keep an aircraft stable for the most part. There are certainly cases where the automation cannot, based on its limited authority (e.g., severe icing), but what is needed is the ability to alert the human to an approaching limit long before a problem occurs and then let the human make a decision regarding what to do.
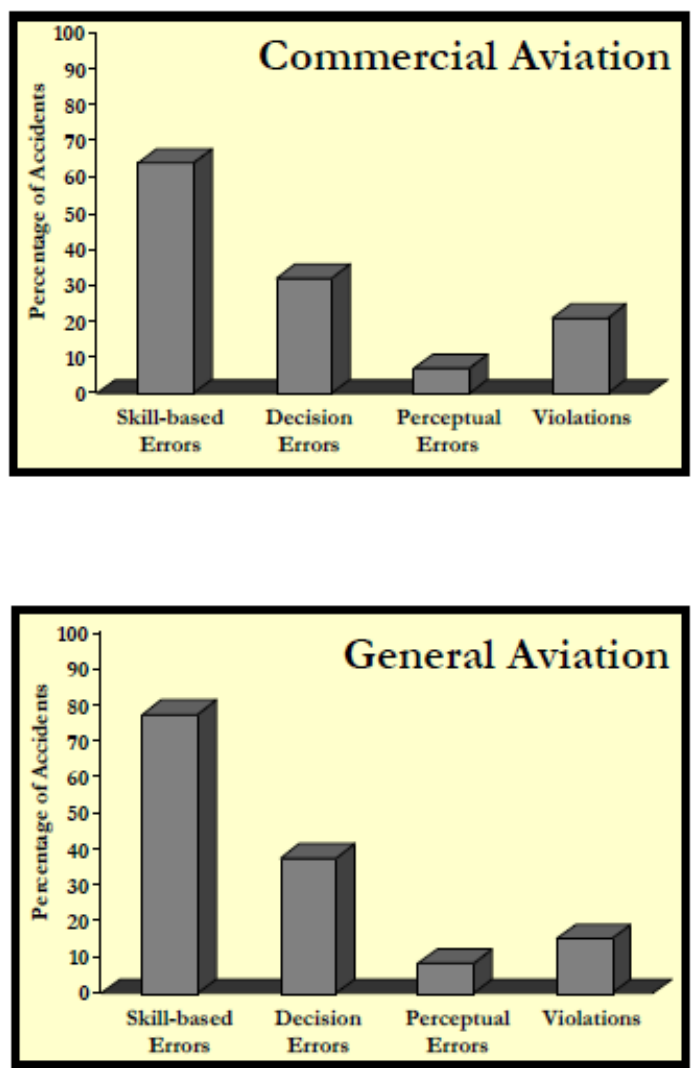

Figure 1. Percentage of commercial and general aviation accidents associated with various human error causal factors ${ }^{16}$

\section{Current Small Aircraft Safety and Ease-of-Use}

Starting from the premise that neither the prospective PAV pilot nor automation, acting alone, can perform the complete task, our goal is to develop a joint human-automation system that can. The requirements are, in part, that the system be safe and easy to use in both initial training and operation. In this section, we briefly review current small aircraft safety and training to identify the feasibility of meeting the goals mentioned earlier (i.e., an order-ofmagnitude improvement in the fatal accident rate, and enabling safe, confident all weather operations with approximately 40 hours of training). We also use this review to indentify the key factors in achieving these goals. 


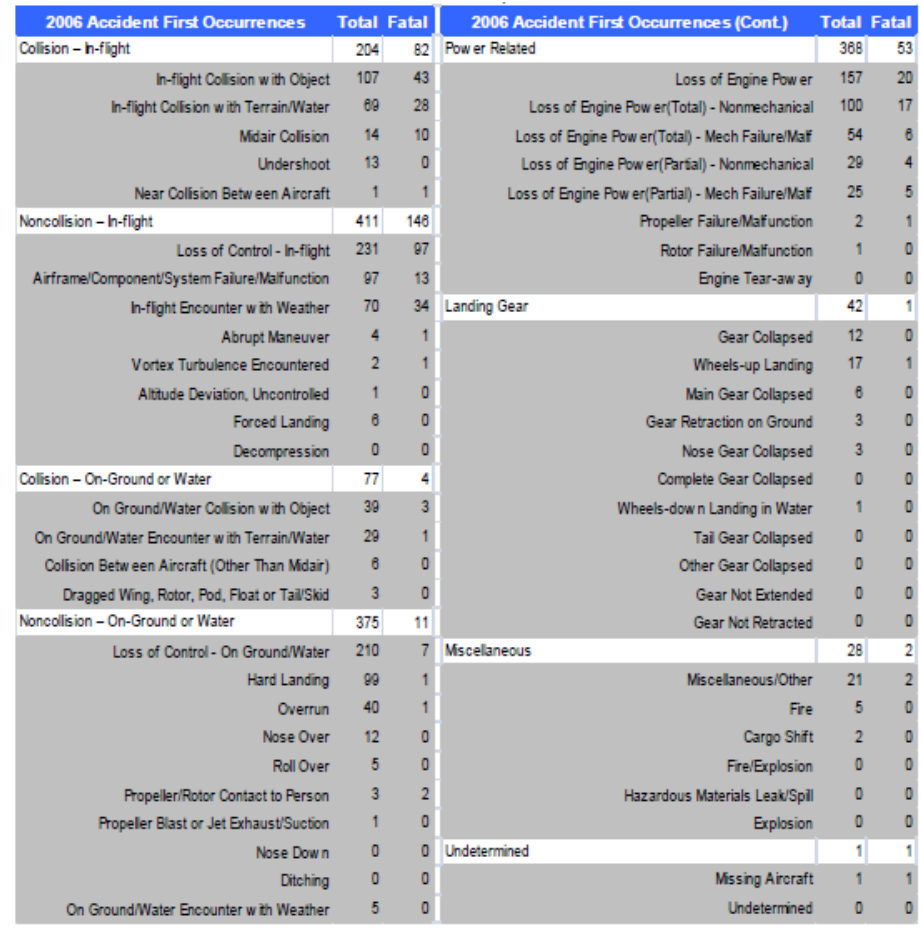

Figure 2. General aviation accident first occurrences, $\mathbf{2 0 0 6}^{17}$
Figure 2 from the National Transportation Safety Board $^{17}$ shows the first occurrences leading to accidents and fatalities for small aircraft in 2006 (the most recently published data). The first occurrence can be considered the first observable link in the accident chain of events. Preventing loss of control and avoiding hazardous weather, terrain, obstructions, and traffic would eliminate approximately $80 \%$ of current fatal accidents. The majority of remaining accidents would then be attributable to loss of power. Given the older engine technology represented in this 2006 data (e.g., the average age of single-engine aircraft in the United States is greater than 30 years), it is reasonable to expect that modern turbine or electronic engines on PAVs could reduce these events by $50 \%$ or more. Together, these items provide a potential order-of-magnitude reduction in fatal accidents relative to current operations. If achieved, this would make travel by personal air vehicle as safe as driving by car. Current technology in the form of terrain and obstruction databases, in-flight weather, cockpit display of traffic, and the ability to display and overlay these items on a multi-function display already provides a solid foundation for hazard avoidance. The key outstanding challenge to achieving the desired increase in level of safety is preventing loss of control.

From a training perspective, fig. 3 presents the approximate percentage of time spent learning various skills to become an instrument-rated private pilot. The total time required to reach this point is approximately 150 hours of flying, consisting of a mix dual instruction and solo practice. This data, collected by Embry Riddle Aeronautical University in the $1990{ }^{\prime}{ }^{18}$, is somewhat dated as it predates the widespread use of the Global Positioning System, introduction of glass-cockpit airplanes, and updated instructional methods such as scenario-based training. The most significant component of training is the amount of effort spent becoming proficient at basic flying skills, i.e., learning how to manipulate the stick, rudder, and throttle to make the airplane go where the pilot wants it with the right attitude and energy, and knowing how it should be flown and positioned for routine operations and select emergencies such as off-field landings. These tasks would be minimally affected by the introduction of glass displays and still require significant attention to master.

Interestingly, multiple simulation studies have investigated the potential benefits of using pathwaybased displays to intuitively indicate where the aircraft should be flown combined with fly-by-wire (FBW) control that dramatically simplifies manual control ${ }^{19,20}$. These studies uniformly show that using automatic control to make the pilot's manual control inceptors (i.e., stick and throttle) directly command the velocityvector of the aircraft allows a non-pilot to perform precision instrument maneuvers, takeoffs, landings with only minutes of training. Figure 4 illustrates how quickly a non-pilot learns to control an airplane through a velocity-vector-based control system. The figure shows lateral tracking error for a untrained, non-pilot flying an extended airport traffic pattern with conventional aircraft dynamics and a simplified, velocity-vector-based dynamics. After overshooting in the first turn ( at $\sim 60$ seconds) the subject maintains

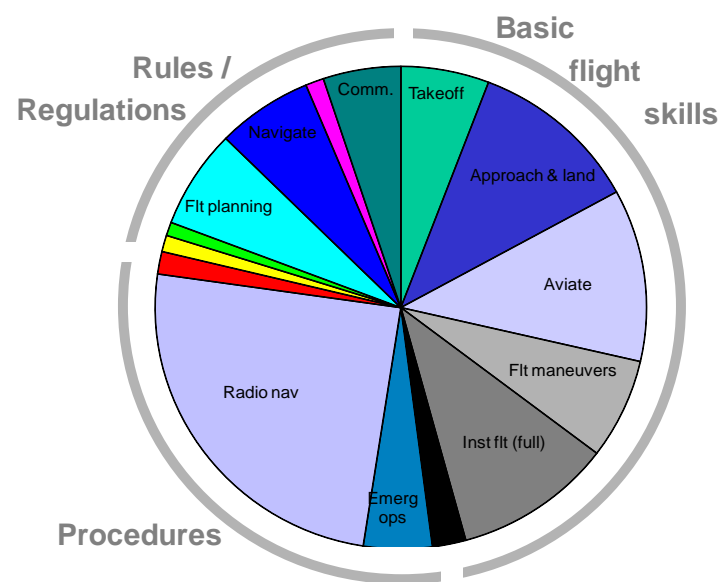

Figure 3. Components of flight training, ab initio to instrument rating. Total-time is approximately 150 flight hours 


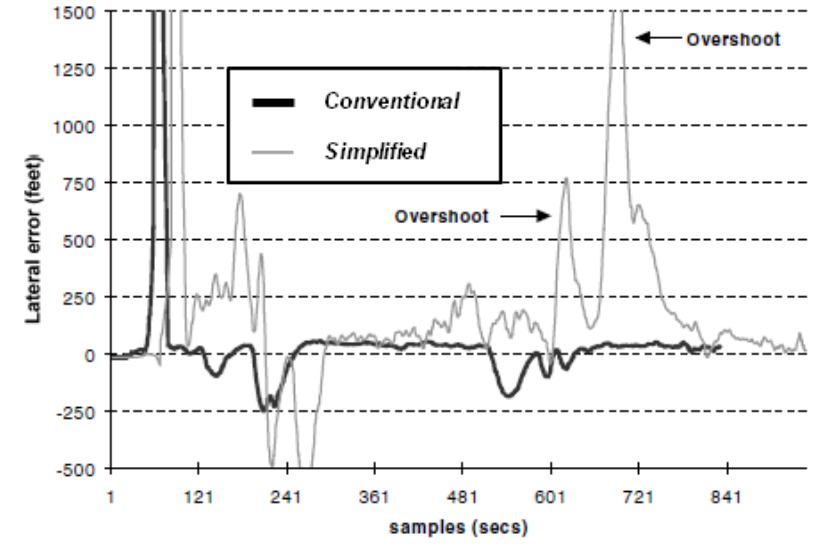

Figure 4. Comparison of conventional and simplified control for non-pilot test subject ${ }^{19}$

lateral tracking within $+/-250$ feet for the rest of the run, even during 90 degree turns. In comparison, the same subject still has trouble controlling the lateral trajectory at the end of the run with the conventional controller, blundering approximately a $1 / 4$ mile off course. In Stewart's study $^{20}$, the participants took off, flew a race track pattern around the runway, and then landed. The task was performed without external visibility above 200'. Of the 7 novice subjects tested, all completed the task on the first run without prior training or practice. After 10 runs all subjects successfully completed the task in the presence of simulated moderate turbulence and a steady 20 knot wind, quartering the runway. If these simulator results could be realized in the field (admittedly a big if), prospective PAV pilots could perform unassisted takeoffs, landings and pattern operations during their first lesson. Rather than extensive training and practicing of cross wind landings, slow flight, turns around a point, basic instrument flight, and practice approaches to internalize the low-level perceptual motor skills, more training could focus on the higher-level judgment and decision making important to non-normal situations. Another significant opportunity for reduced training, and simplified operations, is streamlining the number of procedures in use as experience is gained with Required Navigation Performance (RNP) and NextGen operations. For example, presently there are numerous instrument navigation aids and associated approach procedures in use (e.g., NDB, VOR, LOC, VOR/DME, RNAV, LNAV/VNAV, LPV, ILS, ILS Backcourse, RNP) and instrument-rated pilots need to be prepared to use some number of them. In contrast, RNP navigation is independent of specific navigation sensors and when mature should allow a single, standardized approach type to be used to all runway ends. Similarly, NextGen, or more likely its descendents, may streamline air traffic management and traffic separation procedures so that difficult, time-pressured voice communication fades away and is replaced by something less burdensome to the pilot and that also simplifies the coordination of very high-density operations. At some point, separate visual and instrument flight rules may disappear and be replaced by a single set of rules used regardless of external visibility.

Finally, in the NextGen environment, information needed for making decisions will be generally more complete and easier to obtain compared to today's environment which relies on multiple, dissimilar, sources on the ground and in flight. These improvements will reduce the instruction and practice spent learning how to obtain information pertinent to a flight and allow more training on interpretation and decision making. From the perspective of CTTM, the awareness of routine hazards (e.g. traffic, terrain, obstructions, weather) and factors of flight (e.g., local airport status include runways in use, pattern direction and winds) afforded by the automation is expected to be nearly complete.

Given the available data, it is premature to forecast a minimum training time for PAV pilots. However, in reviewing current flight training requirements and predicting the affects of available and emerging technologies, a long-term goal on the order of 40 hours appears to be credible. In the next section, we provide an overview of the HFC concept that seeks to address the safety, ease-of-use, and HAI issues just reviewed.

\section{Haptic-Multimodal Flight Control System}

\section{A. HFC Description}

This section provides an overview of our proposed HFC system for safe, simple and effective CTTM for PAVs. As mentioned in the introduction, the HFC is part of an overall flight deck concept known as the "Naturalistic Flight Deck" (NFD) ${ }^{2}$. As described in detail in reference 1, the inspiration of the HFC is the metaphor of a rider and good, well trained horse. The reader is encouraged to read Flemisch, et al. ${ }^{21}$ for a detailed description of the metaphor as only a brief description is provided here. Key aspects of the metaphor for design are to provide:

- A vehicle with limited, specialized intelligence, sufficient to move safely and appropriately through the environment without human oversight in routine situations

- $\quad$ Shared control with an operator facilitated through haptic-multimodal interaction. 
As applied to aviation, the first aspect suggests an aircraft with the ability to maneuver through airspace without endangering itself (e.g., staying within envelope limits, avoiding hazards) or others. More advanced implementations could even recognize runways (i.e., perceiving boundaries and obstacles rather than relying entirely on a priori data) and autonomously takeoff and land, albeit usually at the command and supervision of the on-board pilot. It also suggests some degree of independent will if moving toward a self-perceived threat. Limited intelligence implies that the aircraft, like a horse, knows the near-term actions desired by the pilot but does not know about their long-term intentions (other aspects of the NFD support longer-term,

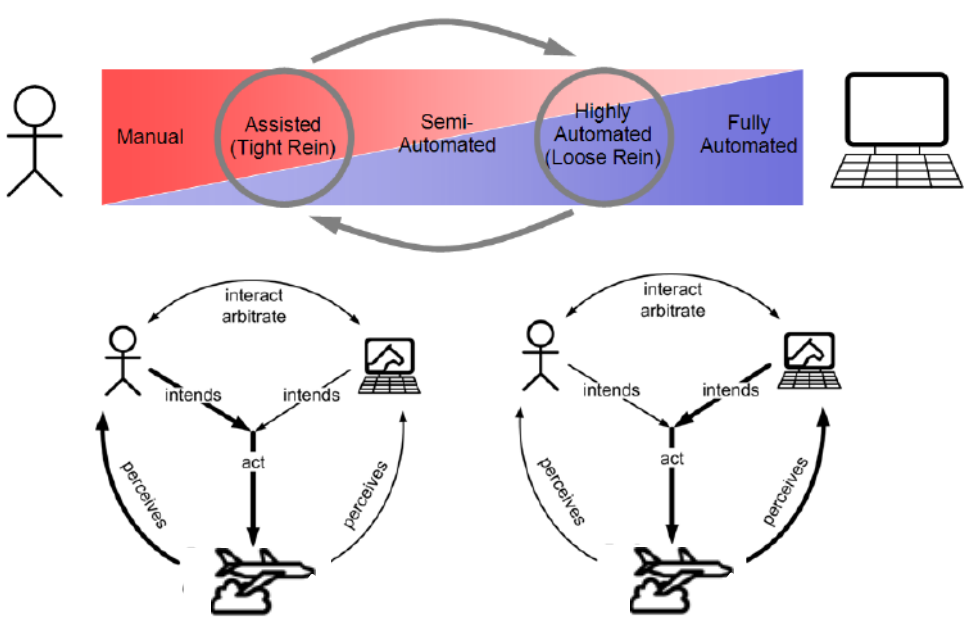

Figure 5. Automation spectrum with the two levels of support, Tight-rein and Loose-rein (above) with different influence of pilot and automation on the aircraft behavior (below) ${ }^{22}$

flight management tasks). The pilot uses simple interactions to keep the aircraft on the desired path when significant trajectory changes are required. If the pilot misses an expected action, other aspects of the NFD support pilot monitoring, alerting, and reengagement. In an advanced implementation, if the aircraft perceives an imminent threat and the pilot is not engaged, it should transition to a more cautious posture, shifting its bias toward safety. Ultimately, it would perform a precautionary, autonomous landing at some point if the pilot remained nonresponsive. This situation should probably be considered an emergency, allowing special handling in the airspace and discouraging abuse of this back-up capability.

The concept of shared control implies that neither the pilot nor vehicle can hand over all aspects of the CTTM to the other agent. Currently, there are two primary modes of operation known as "tight" and "loose" reins. As shown in fig. $5^{22}$, tight-rein corresponds to a form of assisted manual control and loose-rein a form of highly-automated control. As explained shortly, the assisted manual control is provided by a flight-critical fly-by-wire system that supports all other control modes. In tight-rein, higher-level automation functions nominally remain passive, but if hazards are approached they generate forces (i.e. pushes back) on the pilot's hand controls consistent with a preferred avoidance maneuver.

The highly-automated control mode differs from cHAA in two fundamental ways. The first, as already mentioned, is that the automation cannot be coupled to an entire flight plan and requires pilot involvement at significant junctions. The second is that all automation support is managed through haptic and visual elements integrated into the primary control interfaces (i.e., force-feedback stick and throttle) and the displays (a synthetic vision based primary flight display (PFD) and tactical navigation display (TND)). Both of these features have been evaluated in part-task simulation studies and the results are summarized in an upcoming section.

A simplified system diagram of the HFC system is shown in fig. 6. The inner-loop control simplifies the dynamic response of the aircraft, providing predictable control and envelope protection for both the pilot and outer-loop automation. As described in the earlier safety and training overview, simplified flight dynamics combined with envelope protection are central factors in achieving improved safety and ease-ofuse. Implementing these functions is probably best done through a FBW

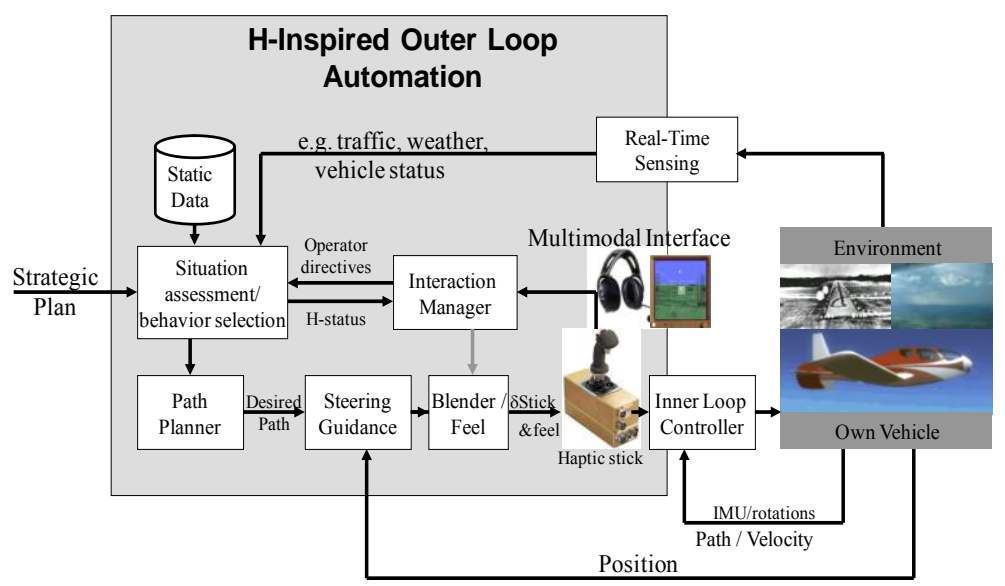

Figure 6. System diagram of HFC 
control system. While FBW systems are certified on current civil aircraft, these implementations all have failure modes that revert to the pilot essentially flying the bare airframe. This sort of reversion is, of course, unacceptable for a FBW PAV. Developing a certifiable FBW system that continues to provide simplified dynamics and protection against all failures that are not "extremely improbable" is a major technical challenge of our concept. That noted, this task should be far easier than developing and certifying a fully autonomous system. It should also be noted that many of the benefits of the HFC in terms of improved human-automation interaction and envelope protection are possible on non-FBW aircraft, albeit with increased training because of the more complex flight dynamics.

The active, force-feedback control interfaces, represented by the control stick in fig. 6 , provide the central point of contact between the pilot and automation. In our current implementation, displacement of these interfaces is the sole connection between the outer- (i.e., assessment, path planning and autopilot) and inner-loop (stability augmentation) control systems. The practical effect of this implementation is that both the pilot and automation have to apply forces to the stick to affect maneuvers. If only one agent applies force, the resulting stick displacement provides observable kinematic feedback to the other. If both agents apply force, differing intentions can be intuitively felt through the proprioceptive interaction. The immediate response of the airplane is determined by the control displacement resulting from the net force input. By increasing or decreasing the forces used by the HFC, its "strength of will" can be modulated between suggestive and dominant. When using light or moderate forces, the pilot can overpower the automation and retain command authority. As described below, the HFC uses its sensing of pilot-applied forces, combined with simple interpretations of the flight situation, to modify or change its actions to better support the pilot.

The outer-loop or automatic guidance functions of the HFC are activated and managed through the primary controls and displays in a manner that leverages the skills and interactions the pilot uses for manual flight. A more detailed explanation is available in ref. 23, but the general principles are summarized here, as are some extensions made since ref. 23 was published. The PFD and TND (fig. 7) display spatial representations of the external environment, overlaid by basic flight parameters. The depiction of the environment includes physical elements like terrain, obstructions, weather, and traffic. The depiction also includes virtual elements like published airspace features (e.g., fixes and airways) and, if present, the planned route of flight. The pilot can direct the HFC to couple to one of these displayed paths using a set of simple interactions described in the next few paragraphs.

If the pilot is free maneuvering (i.e., flying in tight-rein) and wants to direct the airplane to join one of the displayed paths (i.e., transitioning to loose-rein) he can do this two ways. The first method, called "Point to Program" (P2P), is shown in fig. 7. To perform a P2P coupling, the pilot maneuvers the airplane so it is aimed at the desired intercept point. The HFC indicates its recognition of situation by highlighting the targeted segment (fig. 7a). If the pilot pulls the trigger on the stick while the HFC is in this armed state, the HFC generates, displays, and couples to an intercept path onto the targeted segment (fig. 7b). A second button on the stick is used to cycle through and select the desired path if more than one path falls within the targeting criteria as shown in figure 7a. The second method of joining a displayed path is to manually maneuver into proximity and alignment with it. The HFC recognizes this geometry and couples automatically.

Leaving a path and returning to tight-rein is done by either making a sustained maneuver away from the path or pressing a third button the stick. Once established on the path, along-path trajectory junctions (i.e., forks in the road) can be selected using the same button used earlier to select from multiple P2P options. The aircraft's altitude can be managed independently from the nominally displayed path altitudes on the PFD. This is done using a hat switch on the stick to create and set a "designated altitude". With a designated altitude active, offsets of situationally appropriate paths are displayed at this altitude and available to the HFC. The pilot must manually initiate the climb or descent toward the offset, but the HFC levels off when the target altitude is reached. During this transition, the HFC continues to follow the lateral definition of the path.

Functionality similar to an airline autopilot mode-control panel is supported using a second type of path know as a "track-hold." The HFC generates, displays, and couples to track-hold mode if the pilot pulls the trigger to initiate a loose-rein transition when no path is targeted for a P2P. The HFC creates the track hold along a linear extension of the aircraft's velocity vector when the trigger is pulled. The resulting path maintains a constant ground track and inertial flight path angle. Designated altitudes can be used to manage altitude while traveling along a track hold as described earlier. When operating in track-hold mode, the HFC monitors pilot-applied forces. Larger force inputs cause the HFC to transition back to tight-rein. Moderate pilot forces cause the HFC to update the heading and angle of the track hold as the aircraft maneuvers. This works similar to airline autopilot "control wheel steering," allowing the pilot to make path adjustments without having to go back into tight-rein. It is also possible to use P2P intercepts from a track-hold to join displayed segments. 
The haptic power lever allows integrated management of power, speed, and temporal functions through this single interface. As currently implemented, the angle of the power lever represents the power (or thrust) command to the engine controller. By pulling the trigger on the throttle, the HFC activates an auto-throttle mode, back driving the power lever as needed to maintain commanded airspeed. When first activated, the commanded speed is the current airspeed. The commanded value is updated when the pilot applies force to the power lever. The airspeed command is changed at a rate proportional to the pilot's force.

Higher-level, speed-related modes can be managed using switchology similar to what was described for the stick. For example, when coupled to a path, a time of arrival control mode can be armed via a second button press on the power lever. From this condition, a hat switch is used to step-through designated, along-path, metering fixes. If the pilot pulls the trigger on the power lever from this armed condition, the time of arrival control mode engages,

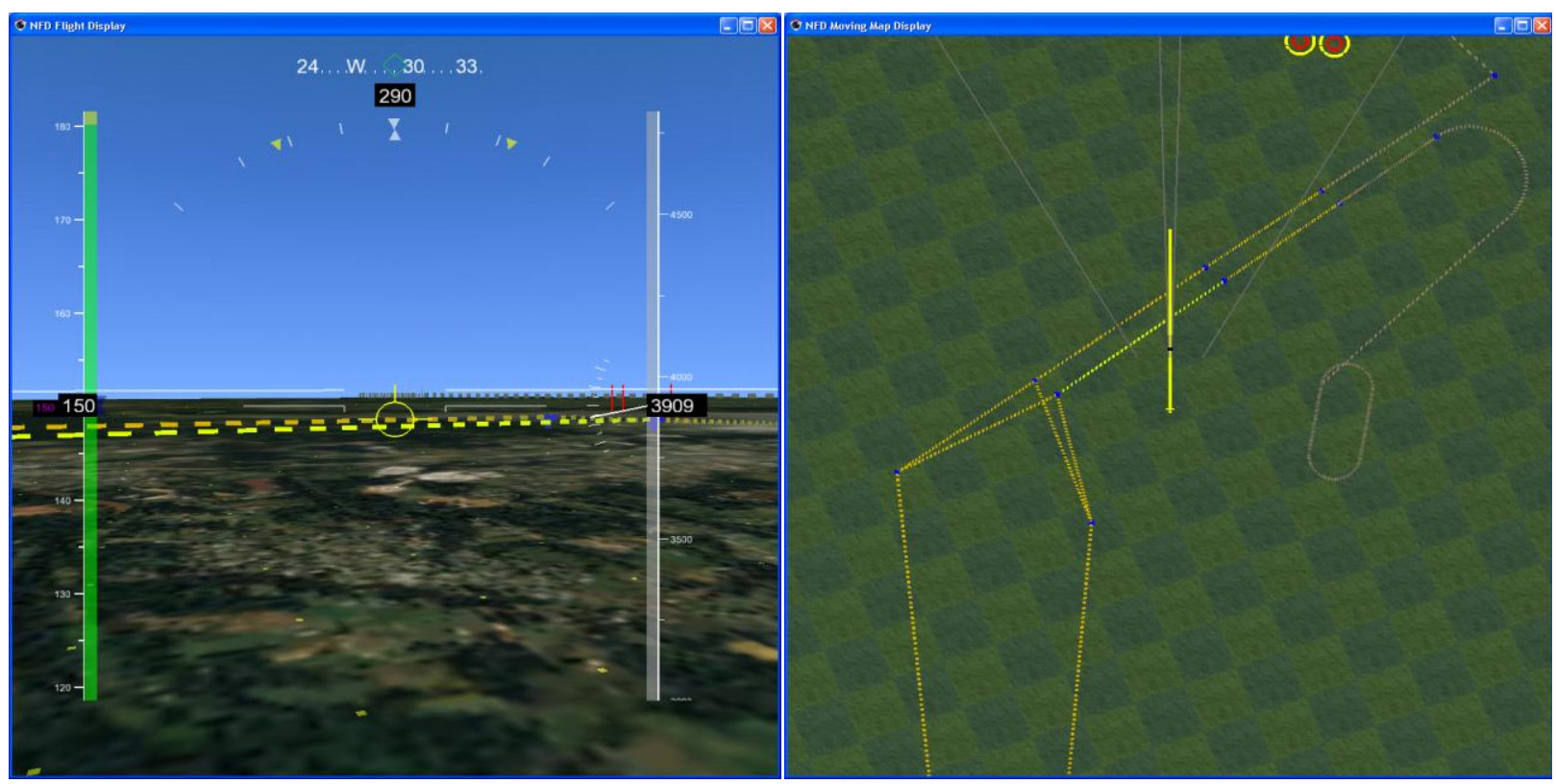

Figure 7a. Displays just prior to performing P2P to join a published approach segment

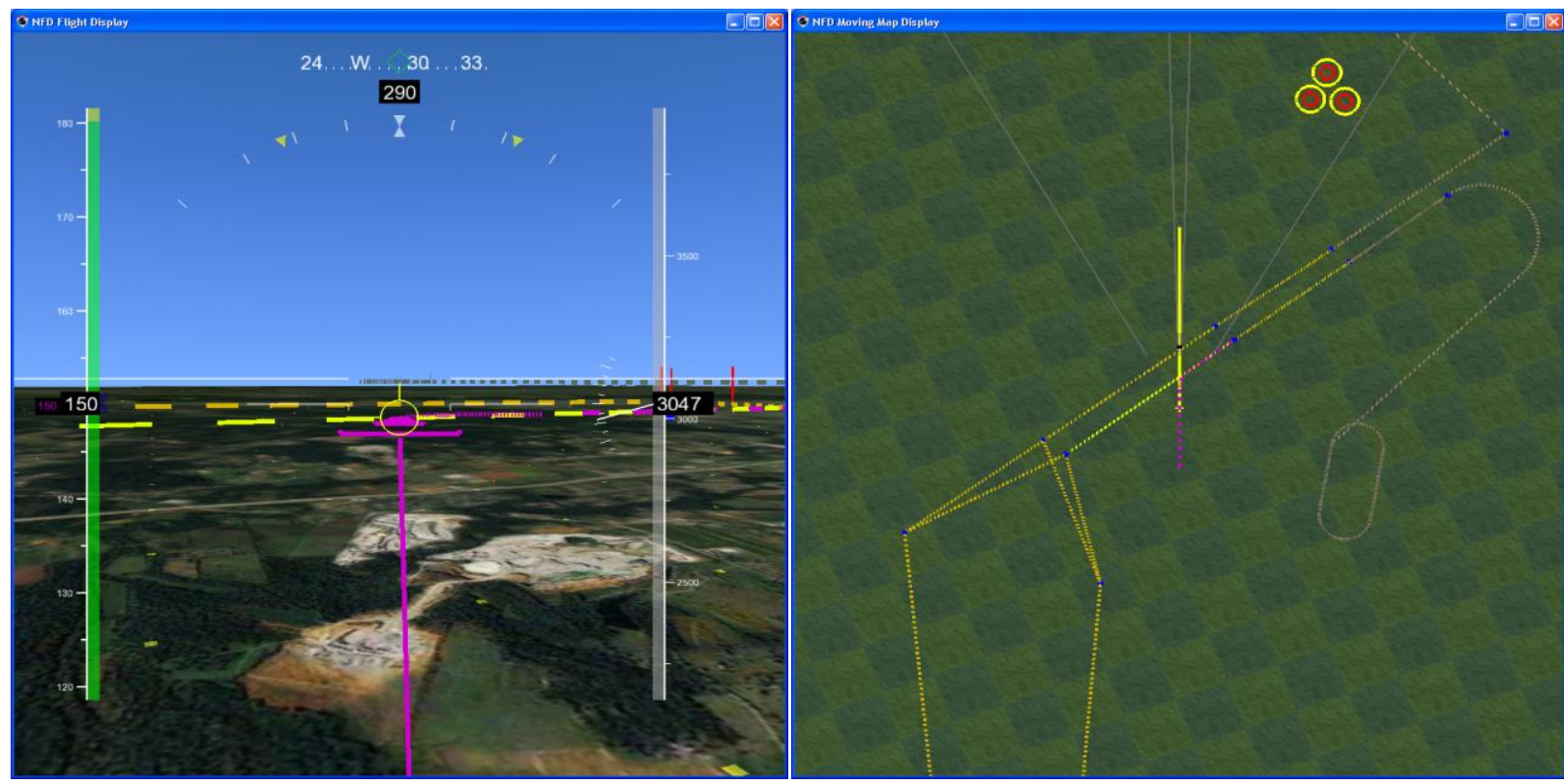

Figure 7b. Displays just after completing P2P 
using the current estimated time of arrival to the fix as its target. Subsequent moderate pilot forces on the power lever are used to change the target time of arrival in a manner similar to managing airspeed.

In the next section, we summarize the results from two preliminary, pilot-in-the-loop simulation studies evaluating the effects of the HFC on task performance, workload, and awareness.

\section{B. Initial Human-in-the-Loop Evaluations}

This section summarizes some of the key findings from two initial evaluations of the HFC in piloted simulation studies. The first study evaluated the usability of the HFC in still and turbulent air. The second study assessed pilot acceptance of the HFC compared to that of conventional interactions. It also assessed HFC's influence on pilot workload, situation awareness and task performance compared to conventional interactions. In contrast to the theme of the paper, we've used moderately experienced, general aviation pilots as subjects rather than non-pilots in these studies. There are several reasons for this. First is the reduced training and improved consistency of subjects who are already familiar with flying. Second, these subjects are familiar with current technology and can provide comparative feedback based on their experiences. Finally, the initial application of many concepts from the HFC is likely to be on current light aircraft rather than PAVs.

\section{Usability Assessment}

The first study ${ }^{23}$ evaluated the basic usability of the HFC in calm and turbulent conditions. A particular concern was whether turbulence would make the $\mathrm{P} 2 \mathrm{P}$ and proximity-join concepts impractical. To evaluate this possibility subjects exercised the elemental automation management tasks supported by the HFC in three conditions in a motion-based simulator at NASA Langley. The three conditions were: 1) cockpit motion on, turbulence off; 2) cockpit motion off, turbulence on; 3) cockpit motion on, turbulence on. The fourth possible combination, motion and turbulence both off was found to be equivalent to condition 1 during dry runs and was not included in the test in the interest of efficiency. Condition 2 is of interest to unmanned applications during which the vehicle could be subjected to turbulence but not the operator. Because the task elements effectively involve manual control tasks, Cooper-Harper Ratings (CHR) ${ }^{24}$ were found to be an appropriate and effective tool for assessing subjective performance and workload. Figure 8 shows a subset of the CHR results from ref. 23. The overall results of this study indicate that the five test subjects generally found the HFC easy to use. In turbulence (with and without motion), the handling qualities ratings typically increased slightly (probably indicating higher workload), which is to be expected. Subjectively, the subjects did not consider this increase to be objectionable and generally less than might be expected with conventional automation interfaces in similar turbulence. All five subjects rated the difficulty of learning to use the HFC as either easy or very easy. It should also be noted that after this study was conducted, significant refinements have been made to further improve the usability of the HFC.

\section{Evaluation of Acceptance and Comparison with Conventional Automation}

The second experiment evaluated pilot acceptance of the HFC concept relative to cases with "conventional" automation and with no automation. The study also assessed the influence of these automation levels on pilot workload and situation awareness. The results of this study will be documented in more detail in a future report and are summarized here.

At first glance, the haptic, single-point-of-control concept can appear to be a technological step backwards. In a modern flight deck, the pilot rarely has to touch the control inceptor. The use of the control inceptor to fly the aircraft is virtually the hallmark of unautomated aircraft. Won't operators consider this to be a step in the wrong direction? After all, use of a manual inceptor may increase their workload in some situations.

We performed an experiment that tested whether pilots preferred the more highly automated approach. In this experiment, 24 instrument-rated pilots with little or no glass cockpit experience but fairly high number of flying hours were tested. Subjects were asked to plan a route in a fictitious airspace, enter that route into a graphical flight planner (virtually connecting the waypoints from point A to point B), and then to fly that route (based on guidance from the graphical flight planner) in a desktop simulator. Each pilot experienced three flight control conditions: Manual (where they flew the simulator using the side stick with no support from automation), Automated (where the aircraft flew itself based on the plan in the planner), and a subset of HFC called Segment-Based Automation (where the subject used the side stick to maneuver from one published leg to the next; after coupling onto that leg, the HFC automation flew the aircraft along that segment.)

All three conditions had the same guidance and symbology on the PFD and TND. In all conditions, as the aircraft approached a segment change, the subject received a 'rumble strip' vibration in the side-stick and an aural alert. While flying the routes, subjects were given a number of air traffic control-like instructions that required them to fly a parallel path, make a strategic route change, and change runways while on approach. While flying the route, 
Track Hold Task

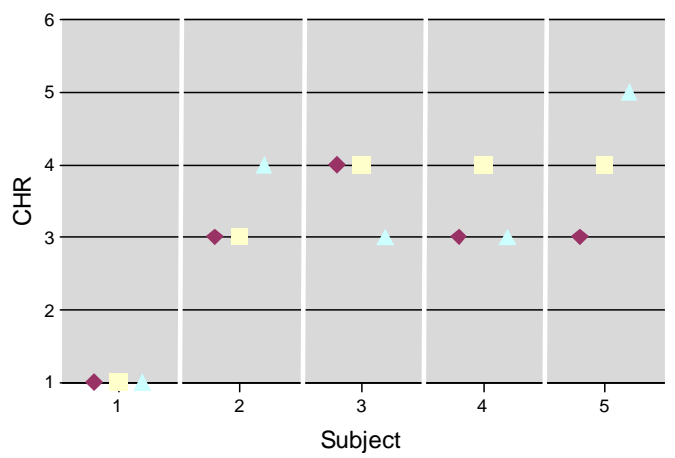

Point to Program Task

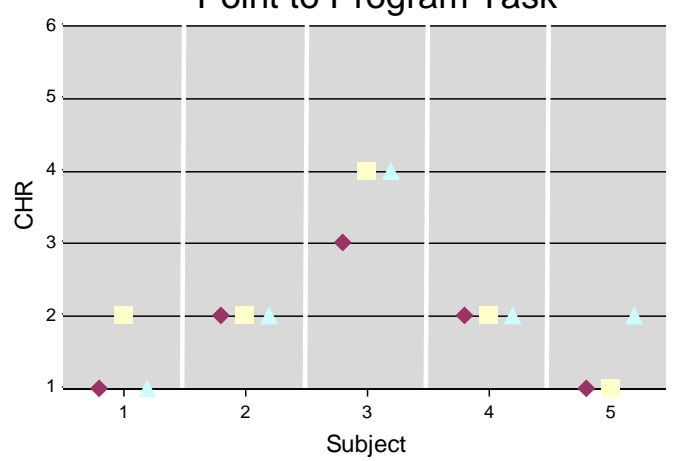

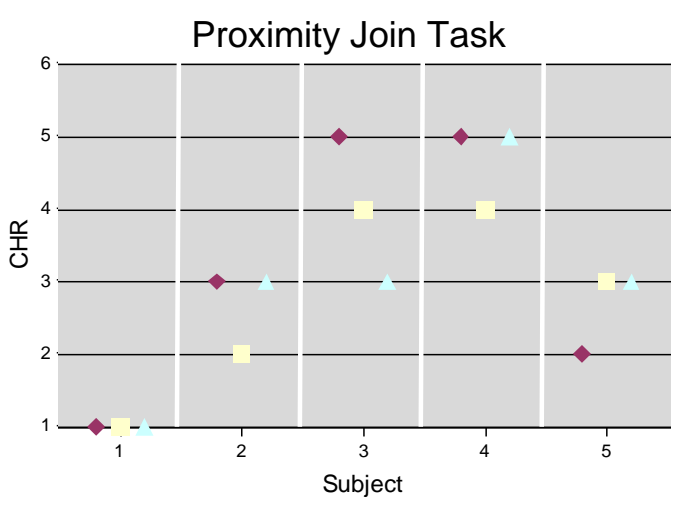

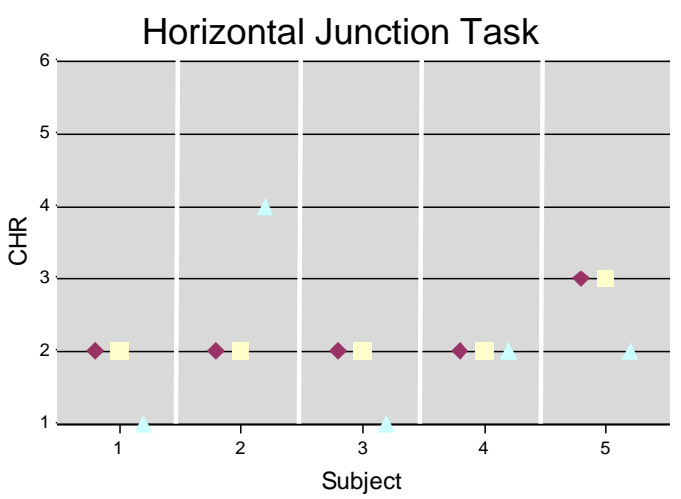

With motion, no turbulence No motion, with turbulence With motion and turbulence

Figure 8. Handling qualities ratings of HFC elemental tasks

the subjects also had two secondary tasks - a self paced math task and a target recognition task. They were told that the flying task was their primary task.

After experiencing all three conditions, subjects were asked to rate their most preferred control mode and their least preferred. They were asked to consider their preference with regard to all three tasks together and to just the flying task. The results showed that pilots overwhelmingly preferred the Segment-Based Automation over the Manual and the Automated conditions. This was true for both the flying task by itself (fig. 9a) and the combined set of tasks (fig. 9b). These results were statistically significant ( $p<0.01$, where $p$ is the probability of obtaining the observed effect under a 'null hypothesis'). What was also interesting was that their responses to the least preferred method of control were split between the Manual condition and the Automated condition (fig. 10). This indicates that neither the Automated nor the Manual is a clear second place favorite. A fully automated flight deck would not be preferred by the majority of the subjects.

Additionally, subjects were asked to rate their own situation awareness for the three different control conditions. Again, they were asked to consider just their flight situation awareness (e.g., where they were, how fast they were going) and their overall situation awareness (e.g., flight plus how many questions they had answered, where the last target was). Again, the results significantly favored the Segment-Based Automation over the other two ( $\mathrm{p}<0.05)$ for both the flying task (fig. 11a) and the combined task (fig. 11b). Similar to the preference questions, the results were split regarding which condition they felt the least aware of their situation (fig. 12). An attempt was made to objectively validate the subjects' ratings of their own situation awareness. A Situation Awareness Global Assessment Technique (SAGAT) test battery ${ }^{25}$ was developed that probed the subject at random points in the test to ask them situation awareness questions with all the screens blanked out. The probes consisted of asking them two flying questions (e.g., what's the next waypoint? What's your current airspeed? Where are you on the map?) and one target question (e.g., What was the color of the last target? Where was the last target?) and one math question (e.g., how many questions have you answered, what was the last answer you typed in?). There were three SAGAT probes 
during each run and they always asked different questions. The results were not significant. However the numbers were higher for the Segment-Based Automation than the other two. It may be that due to the small number of questions, the SAGAT was simply not sensitive enough to produce a significant result. Figure 14 shows the results for both the flight information and the combined information.

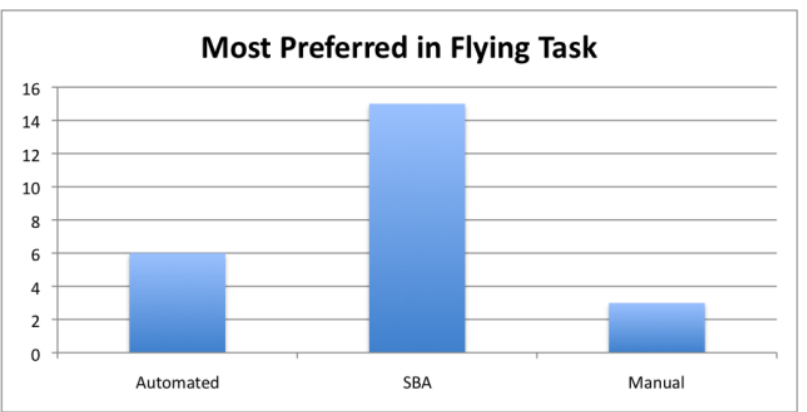

Figure 9a. Most preferred for the flying task

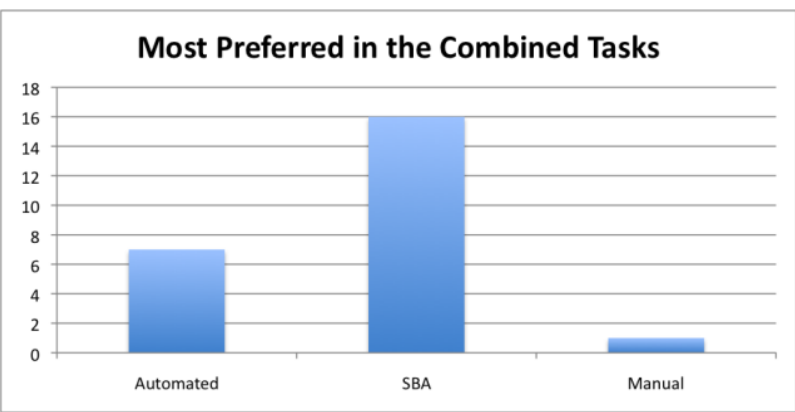

Figure 9b. Most preferred for the combined tasks

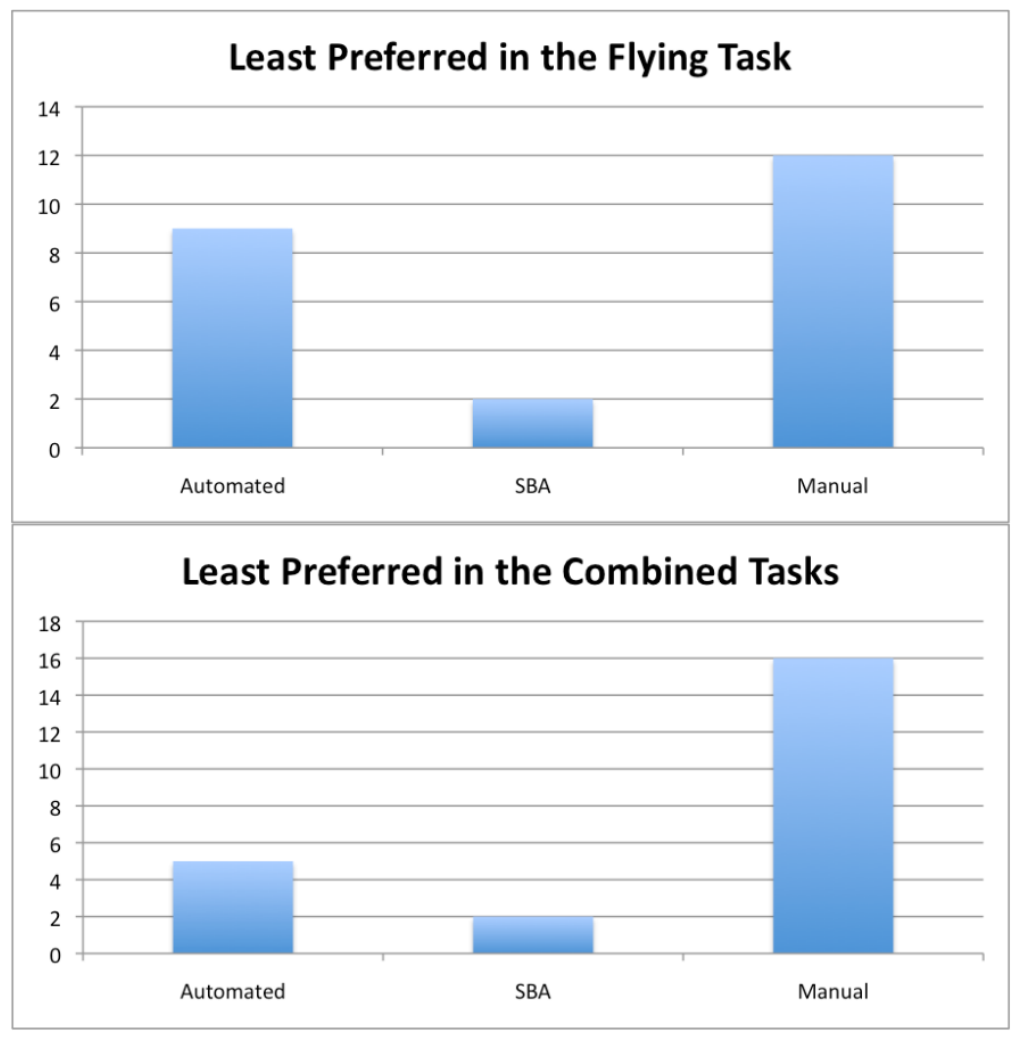

Figure 10. Least preferred conditions 


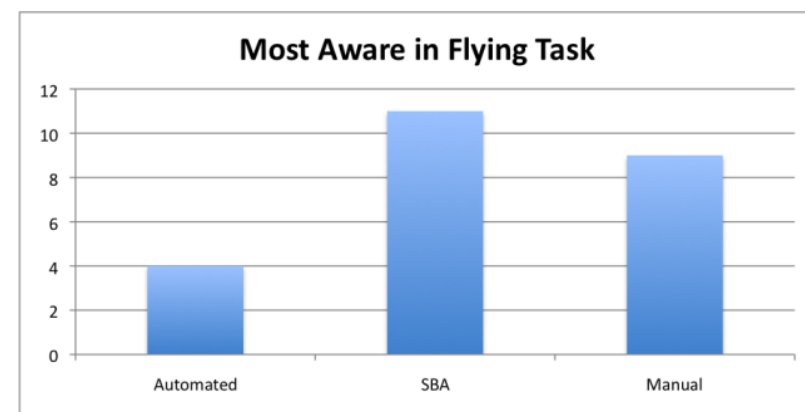

Figure 11a. Highest situation awareness regarding Flying

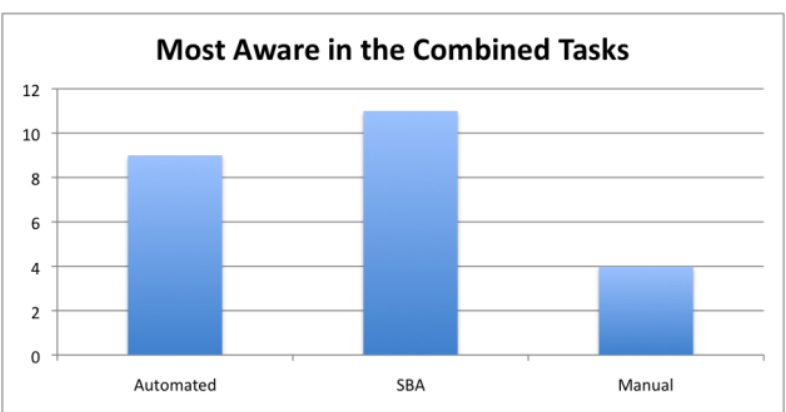

Figure 11b. Highest situation awareness regarding combined tasks

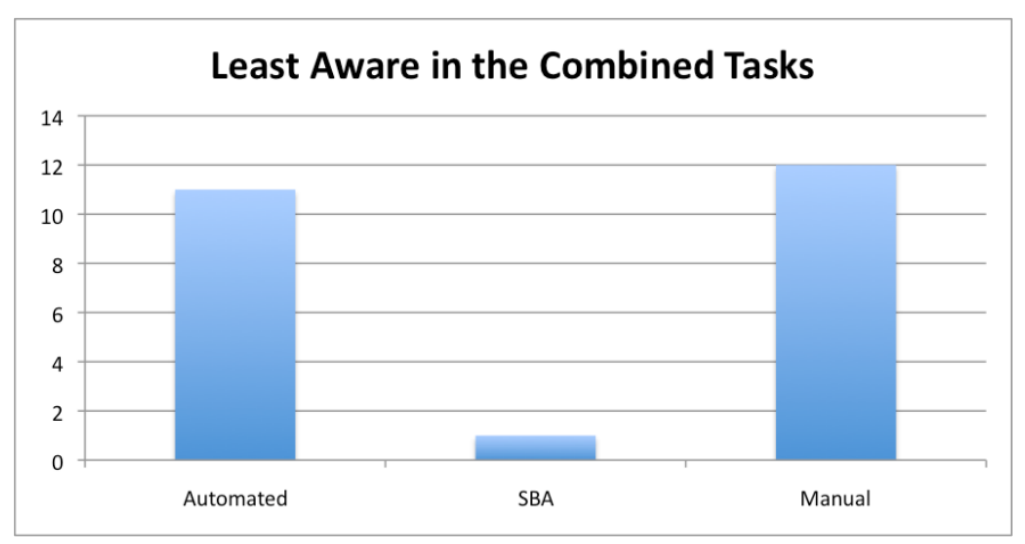

Figure 12. Least situation awareness

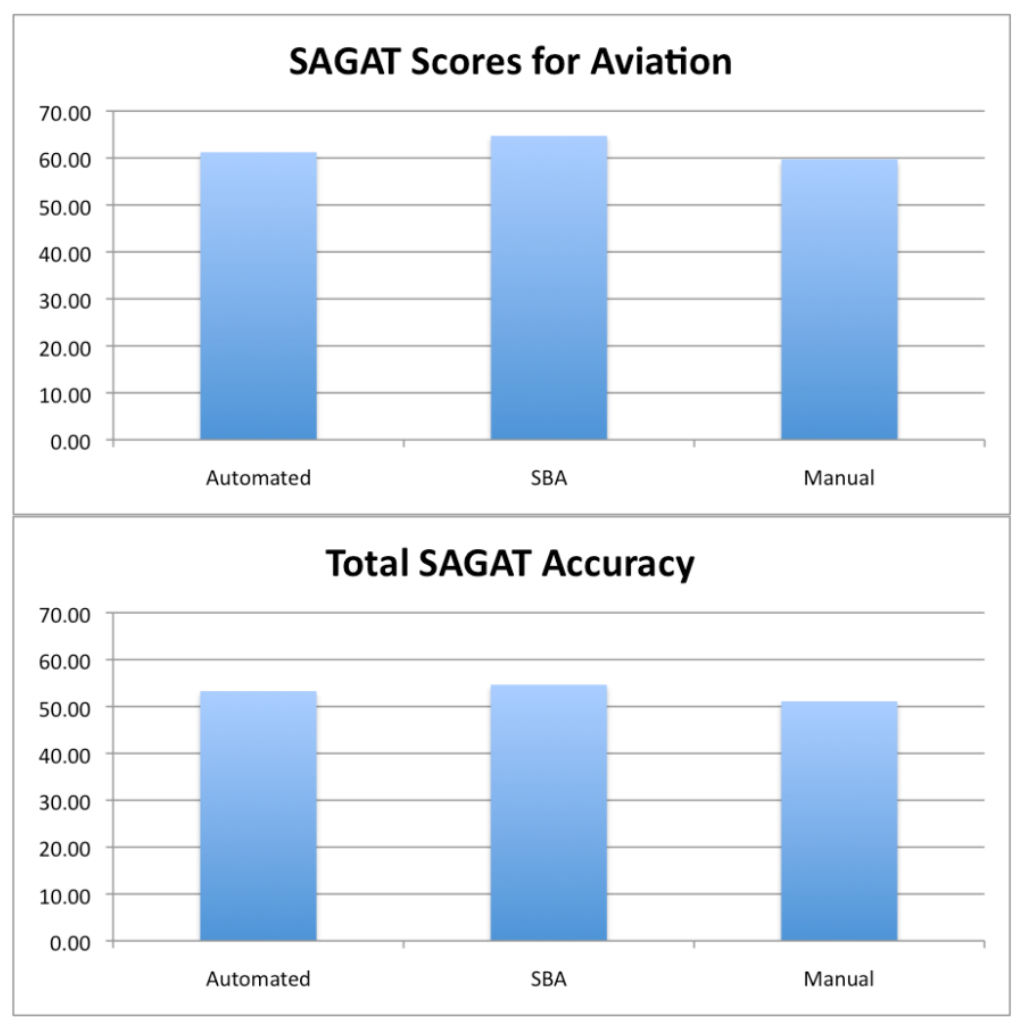

Figure 13. SAGAT results

14

American Institute of Aeronautics and Astronautics 


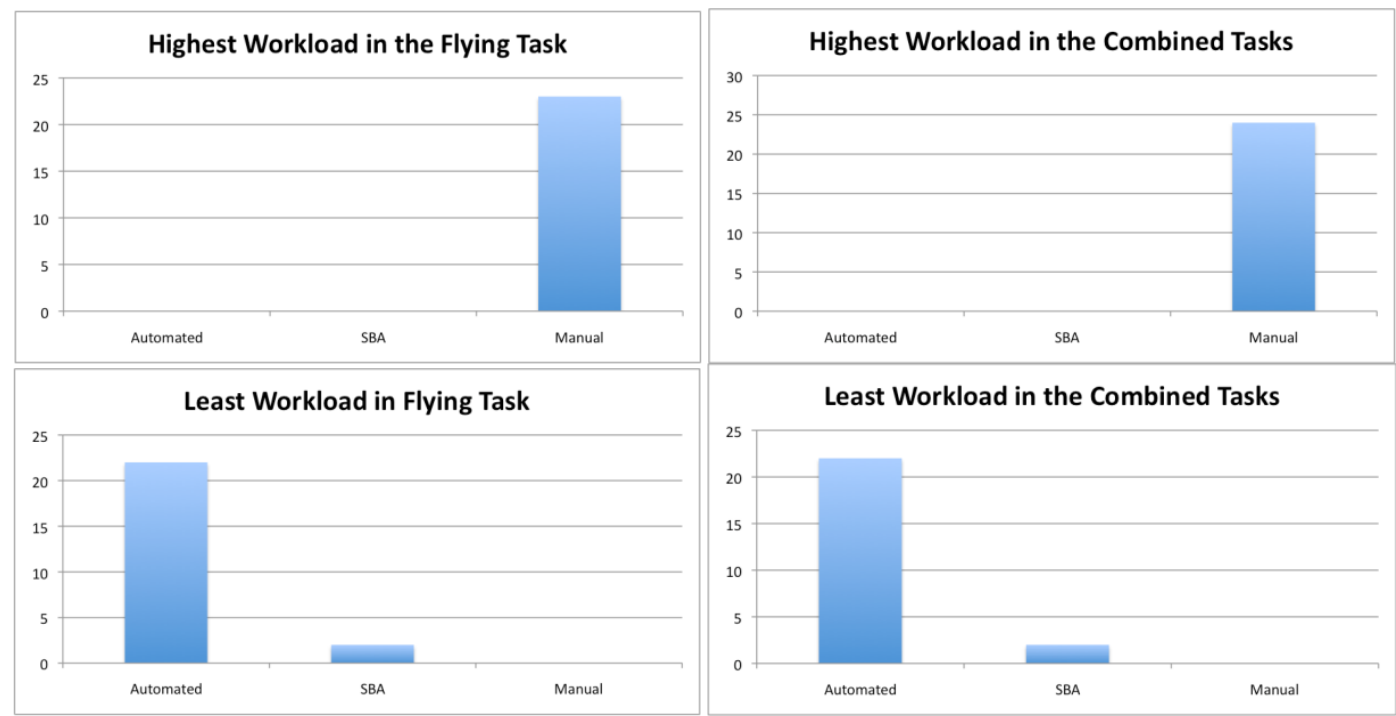

Figure 14. Highest and lowest (i.e., least) workloads

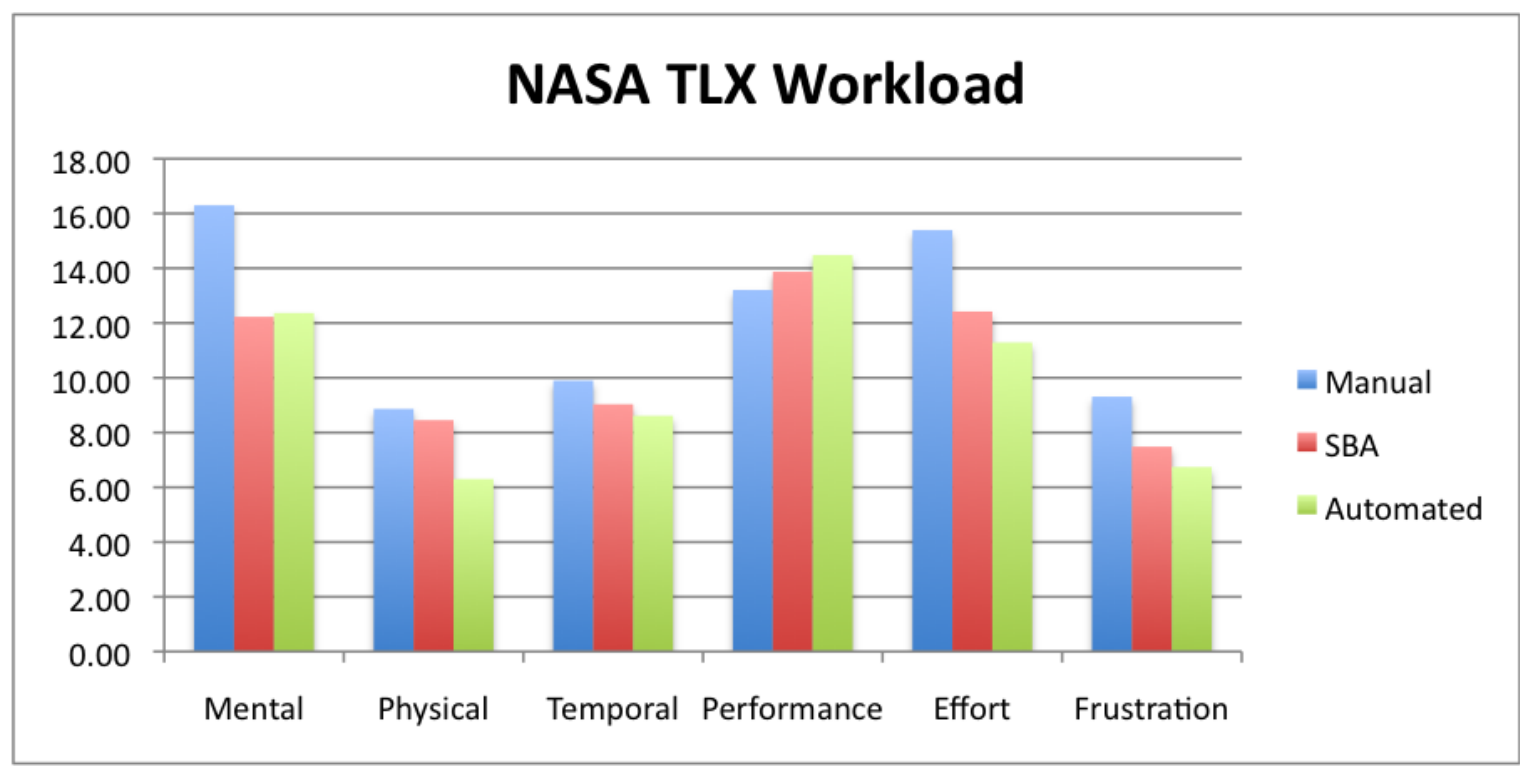

Figure 15. Task Load Index results

Finally, subjects were asked which condition had the least and the greatest workload. The results are not surprising (fig. 14): They rated the Automated as having the lowest workload and Manual as the highest ( $<0.01$ ). What is interesting is that the Segment-Based Automation never rated as highest or lowest. We believe that this represents a 'Goldilocks' spot with regard to workload - "not too much, not too little, just right." We also used the NASA-TLX workload measurement instrument ${ }^{26}$. Statistical significance varied (e.g., for Mental and Effort Workload, the Manual was significantly higher than the Fully Automated or Segment-Based Automation ( $p<0.05)$, but no significant difference between the Segment-Based Automation and Fully Automated; and there was no significance in the differences other differences.) However, the raw TLX data (fig. 15) taken in conjunction with the "greatest and least" data sets further reinforces the 'Goldilocks' claim.

The results of this study indicate that Segment-Based Automation using the HFC concept is not a step backwards, but a significant step forwards. 


\section{Conclusion}

Personal air vehicles (PAVs) that are as safe, reliable, cost effective, and easy to use as cars would give us tremendous new freedom to travel for work and play. While the idea of "everyman's" airplane is as old as aviation, the technology to achieve this dream is close at hand. That said, the technology of contemporary, highly-automated aircraft and emerging autonomous vehicles, by itself, is unlikely to enable passenger-carrying PAVs in the near term. At first glance, these technologies appear to make full-autonomy seem likely in the near future. However at present, even emerging autonomous vehicles rely on specialized, task-specific algorithms that have limited ability respond appropriately in novel situations. Since the environmental and situational complexity of flight virtually assures novel situations will occasionally be encountered, fully autonomous airplanes are probably a distant vision, at least for safety-critical applications like carry human cargo. Further, the conservative nature of aviation regulation and regulators makes it unlikely that technology with sufficient intelligence for fully-autonomous operation could be certified for safety-critical applications without first having significant operational history in less critical applications. These factors lead us to conclude the only credible path to PAVs must retain the human in the pilot's seat and leverage their perception, common sense, general intelligence, and creative thinking. At the same time to enable ease-of-use with improved safety, technology must be used to perform or support tasks requiring swift and precise control, extreme vigilance, extensive memory, and complex computation.

We have applied this philosophy to the development of a haptic-multimodal flight control (HFC) system that supports the pilot in the conduct of control and tactical trajectory management tasks in a way that is easy to learn and use. The HFC provides protection against the most common contributors to accidents and is designed to avoid or mitigate human-automation interaction breakdowns that are common contributors to loss of situation awareness on current highly automated aircraft. The HFC differs from conventional automation in several important ways. First, all control automation is managed and monitored through the primary controls and displays. The pilot skills and actions used to direct the automation are essentially the same as used for assisted-manual flight and workload peaks are avoided by not requiring separate autopilot programming and data entry tasks. Finally, unlike current control automation, the HFC is designed to require regular, but simple, physical involvement by the pilot during the conduct of a flight.

Initial simulation evaluations of the HFC support claims that it is easy to learn and use and that this ease-of-use is largely retained in turbulent flight conditions. In a comparison with manual and fully-automated forms of interaction, it was preferred by a large majority of test subjects and tended to increase subjective awareness ratings of the flying task and the overall set of tasks.

Significant work remains before the full HFC could be applied to PAVs. The most technically uncertain aspect is the reliance on full-time, stability and control augmentation to provide simplified flight dynamics and envelope protection. Flight testing is needed to verify that ease-of-use suggested in simulation is achieved in flight, particularly during takeoff and landing. Also, design and certification criteria are needed for a practical implementation retaining simplified dynamics in the presence of subsystem and component failures not shown to be extremely improbable. Other aspects of the HFC, in particular the integration of automation management into the primary controls and displays, could be applied to current, highly-automated aircraft and have the potential to significantly improve the safety and usability of these aircraft.

\section{References}

${ }^{1}$ Billings, C. E., Aviation automation: The Search for a Human-Centered Approach, Lawrence Erlbaum Associates, Mahwah, NJ, 1997.

${ }^{2}$ Schutte, P. C., Goodrich, K. H., et al, "The Naturalistic Flight Deck System: An Integrated System Concept for Improved Single-Pilot Operations," NASA/TM-2007-215090, 2007.

${ }^{3}$ Bjork, L., Piloting Basics Handbook McGraw-Hill Professional, New York, 2001.

${ }^{4}$ Orasanu, J., Martin, L., Davison, J., "Errors in Aviation Decision Making: Bad Decisions or Bad Luck?," Fourth Conference on Naturalistic Decision Making, Warrenton, VA, May 1998.

${ }^{5}$ Belcastro, C., Foster, J., “Aircraft Loss-of-Control Accident Analysis,” AIAA-2010-8143, 2010.

${ }^{6}$ Moore, M., “Aviation Frontiers: On-Demand Aircraft,” AIAA-2010-9343, 2010.

${ }^{7}$ Joint Planning and Development Office (JPDO), "Concept of Operations for the Next Generation Air Transportation System Joint Planning and Development Office I Version 3.2Concept,” 2010.

16

American Institute of Aeronautics and Astronautics 
${ }^{8}$ Aircraft Owners and Pilots Association (AOPA), “The Flight Training Experience,” AOPA, Fredrick MD, 2010.

${ }^{9}$ AOPA Air Safety Institute, “2010 Nall Report,” AOPA Air Safety Institute, Fredrick MD, 2011.

${ }^{10}$ Gilmore, M. "RQ-4B Global Hawk Block 30 Operational Test and Evaluation Report,” Director, Operational Test \& Evaluation (DOT\&E), US Department of Defense, May 2011.

${ }^{11}$ Williams, K., “A Summary of Unmanned Aircraft Accident/Incident Data: Human Factors Implications,” Federal Aviation Adminstration, 2004.

${ }^{12}$ Voelcker, J., “Autonomous Vehicles Complete DARPA Urban Challenge,” IEEE Spectrum, November 2007.

${ }^{13}$ Markoff, J., “Google Lobbies Nevada to Allow Self-Driving Cars,” New York Times, May 10, 2011, pp. A18.

${ }^{14}$ Shepardson, D., "NHTSA chief skeptical of Google's driverless vehicles,” Detroit News, June 12, 2011.

${ }^{15}$ Scherer, S. Singh, S., Chamberlain, L., Elgersma, M., "Flying Fast and Low Among Obstacles: Methodology and Experiments," The International Journal of Robotics Research, Vol. 27, No. 5, May, 2008, pp. 549-574.

${ }^{16}$ Wiegmann, D., Rantanen, E., "Defining the Relationship Between Human Error Classes and Technology Intervention Strategies," Aviation Human Factors Division Institute of Aviation, rept. AHFD-03-15/NASA-02-1, University of Illinois at Urbana-Champaign, 2003

${ }^{17}$ NTSB, “Annual Review of Aircraft Accident Data U.S. General Aviation, Calendar Year 2006,” National Transportation Safety Board, 2006.

${ }^{18}$ Hampton, S., "Training Issues Associated with the Advanced General Aviation Transportation Experiment and the Potential for Enhanced Decision-Making Using New Cockpit Displays,” IEEE Paper No. 0-7803-6395-7/0, 2000.

${ }^{19}$ Beringer "Applying Performance-Controlled Systems, Fuzzy Logic, and Fly-By-Wire Controls to General Aviation," DOT/FAA/AM-02/7, 2002.

${ }^{20}$ Stewart, E., “A piloted simulation study of advanced controls and displays for novice general aviation pilots," AIAA-94$0276,1994$.

${ }^{21}$ Flemisch, F. O., Adams, C. A., Conway, S. R., Goodrich, K. H., Palmer, M. T., \& Schutte, P. C., "The H-metaphor as a guideline for vehicle automation and interaction," NASA/TM-2003-212672, 2003.

${ }^{22}$ Flemisch, F., Kelsch, J., Loper, C., Schieben, A.., “Automation spectrum, inner / outer compatibility and other potentially useful human factors concepts for assistance and automation,” DLR German Aerospace Centre Braunschweig, Germany, 2008.

${ }^{23}$ Goodrich, K., Williams, R., Schutte, P., "Piloted Evaluation of the H-Mode, a Variable Autonomy Control System, in Motion-Based Simulation," AIAA-2008-6554, 2008.

${ }^{24}$ Cooper, G.E., and Harper, R.P. Jr., "Use of Pilot Rating in the Evaluation of Aircraft Handling Qualities," NASA TN D$5153,1969$.

${ }^{25}$ Endsley, M.,"Direct Measurement of Situation Awareness: Validity and Use of SAGAT," Situation Awareness Analysis and Measurement, Lawrence Erlbaum Associates, Mahwah, NJ, 2000.

${ }^{26}$ Hart S. G., Lowell S. E., 1988, "Development of NASA-TLX (Task Load Index): Results of Empirical and Theoretical Research," Human Mental Workload, North Holland Press, Amsterdam, 1988, pp. 239-250. 